Research Article

\title{
Genomic Analysis for Haloacid Dehalogenase in Bacillus megaterium WSH-002
}

\author{
Luqman Arif Zulkarnain*, Fahrul Huyop \\ Department of Biosciences, Faculty of Science, Universiti Teknologi Malaysia, 81310 UTM Johor Bahru, \\ Malaysia
}

Article history:

Submission September 2021

Revised September 2021

Accepted October 2021

*Corresponding author:

E-mail: manarifz@yahoo.com

\begin{abstract}
Bacterial dehalogenation is one of the processes that can reduce environmental pollutions. The attributes of $B$. megaterium that can grow in a polluted environment suggested that its genome contains pollutant degrading genes. To date, there were no reports related to dehalogenase in $B$. megaterium WSH-002 and how it was regulated. Therefore, the presence of environmentally important genes that can detoxify organohalogens in many microbial genomes, including $B$. megaterium WSH-002 will be investigated. The genome sequence of $B$. megaterium WSH-002 was retrieved from NCBI databases. It was then annotated through the RAST server to identify all the putative dehalogenase gene sequences. The selected gene sequence was converted into amino and went through BLASTp via UniProt database. The highest percentage identity of the amino acid sequence to any dehalogenases was subjected to further identification of specific dehalogenase domain using InterPro Scan server. The results from genome annotations have shown its potential for bioremediation due to the presence of putative dehalogenase protein. Only one type of haloacid dehalogenase was identified. It was classified as haloacid dehalogenase type II because its amino acid sequence is highly identical with HAD_type_II and HAD_L2-DEX. The study concluded that the genome of $B$. megaterium WSH-002 contains a haloacid dehalogenase gene that is useful for the biodegradation of halogenated compounds. In the future, further investigation on the expression of the dehalogenase gene as recombinant protein and to study its protein structure and functions will be considered.
\end{abstract}

Keywords: Bacillus megaterium WSH-002, Genomics, Haloacid dehalogenase

\section{Introduction}

Halogenated compounds are widely found in the environments such as lakes, soil, groundwater, and rivers [1]. These compounds can be naturally produced by microorganisms or being produced by chemically synthesised [2]. The synthetic halogenated compound is being produced as active ingredients for the production of herbicides, pesticides, and organic solvents [3-5]. Haloalkanoic acids are one of the compounds widely used in the agriculture industry. It has the properties of being toxic to the environment, which could cause harmful effects to human health [6]. Several human diseases were caused by these organo-halogen compounds, including digestive disorders, oral infections, organ damage, toxic reproduction, skin and respiratory irritations [7, 8]. Malaysia is one of the countries in the Asia Pacific that uses pesticides extensively in many agricultural activities. Approximately 1.5 million hectares of land were used for rubber tree cultivation and 0.6 hectares for oil palm trees [9]. As being studied by Awang and his colleagues [9], the extensive usage of pesticides in agriculture accounted for nearly $50 \%$ of the total number of 5,152 cases of human poisoning in Malaysia. Statistically, the number of these toxic compounds gradually increased from 50 naturally produced compounds in 1968 to more than 5,000 in 2015 and surprisingly still increasing [10].

Microbial dehalogenases have been widely studied and proven to degrade various halogenated compounds [11-13]. These dehalogenases have been grouped into hydrolytic, haloalcohol, and

How to cite:

Zulkarnain LA, Huyop F (2022) Genomic Analysis for Haloacid Dehalogenase in Bacillus megaterium WSH-002. Journal of Tropical Life Science 12 (1): 73 - 82. doi: 10.11594/jtls.12.01.07. 
cofactor-dependent [14]. Haloacid dehalogenase is classified under hydrolytic dehalogenase which hydrolyses haloalkanoic acids and is converted into hydroxyl compound [15]. The process of dehalogenation is designated as the initial step in the degradative pathway and is the most crucial in degrading chlorinated aliphatics. The mechanism involves cleaving the halogen bonds by nucleophilic substitution, replacing the halogen ion with hydroxyl group derived from water. In addition, dehalogenation involves in degradation of chlorinated aliphatic acids such as $\alpha$-chloro substituted of D- and L- haloalkanoates (2,2-dichloropropionate and/or D- and L-chloropropionate) and haloacetates (monochloroacetate, dichloroacetate, and trichloroacetate) [16, 17]. Bacillus megaterium is a Gram-positive bacterium, ubiquitous from soil to seawater, sediment, rice paddies, honey, and dried food [18]. This bacterium was considered an ideal organism for industrial application used for more than 50 years [18]. Plasmids in Bacillus sp. commonly carrying genes involved in the degradation of toxic compounds, biocontrol, antibiotic, and heavy metal resistance gene and are transferable among genus or species [19-22]. Interestingly, $B$. megaterium was able to degrade halogenated compounds as a carbon source through the production of dehalogenase enzymes and deserved further investigation [23-27].

According to World Health Organization (WHO), genomics is defined as studying genes and their functions [28]. Microbial genomes encompass all chromosomal and extrachromosomal genetic material. Microbial genomics in bacteria is considered very diverse [29]. Bacterial genomes usually, consists of a single circular chromosome but some species also contain more than one chromosome, such as in Deinococcus radiodurans, or linear chromosomes such as in Bacillus subtilis strain, or a combination of linear and circular chromosomes such as in Agrobacterium tumefaciens [30-32]. The study of microbial genomes helps us better understand the genetic composition that contributes to their tangible characteristics that can be further utilized for specific functions [33]. For instance, microbial dehalogenation can be seen as interesting potential outcomes for the bioremediation process and from a genomics and bioinformatics perspective. Recent genomic research has been used to identify gene and metabolic pathways, essential for the whole gene expression to investigate the co-expressed genes $[34,35]$.

The current study will ascertain partial genetic organisation related to dehalogenases and their operon to regulate dehalogenases. There are no reports on haloacid dehalogenase in B. megaterium strain WSH-002. However, many literatures reported $B$. megaterium were able to produce dehalogenase-like enzymes and limited studies explained the use of whole-genome sequencing to elucidate genetic sources of pollutant degradation potential $[36,37]$. In the most recent report, $B$. megaterium strain BHS1 was found to contain haloacid dehalogenase type II gene (dehLBHS1) [38] and the strain BHS1 was reported closely related to B. megaterium WSH-002 [39]. Hence, it can be hypothesised that $B$. megaterium WSH-002 contains a dehalogenase gene which is potential for bioremediation. This study screens and partially annotates the whole genome for possible putative dehalogenase genes in $B$. megaterium strain WSH-002. This will shed light in the future on the genetic organisation and regulation of dehalogenases for haloacid degradation.

\section{Material and Methods Genome retrieval and genome annotation}

The genome sequence of $B$. megaterium WSH-002 with the Accession Number of CP003017 was obtained from the National Center for Biotechnology Information (NCBI) databases (www.ncbi.nlm.nih.gov/) and downloaded as FASTA format. The genome sequence of $B$. megaterium WSH-002 was then uploaded in Rapid Annotation using Subsystem Technology (RAST) server (rast.nmpdr.org/) to perform genome annotation [40]. The setting parameter for genome annotation was set as default. After completion, the annotated genome sequence was downloaded as an Excel file (xls).

\section{Screening for putative haloacid dehalogenase gene}

The screening on the genome sequence was to search for the putative haloacid dehalogenase genes. The identified putative haloacid dehalogenase gene was translated into amino acid sequence followed by protein BLAST search using Uniprot databases [41, 42]. For further analyses, the highest amino acid sequence identity to any related dehalogenases will be selected. 
Determining of Protein Domain and Families of Putative Haloacid Dehalogenase

The selected putative haloacid dehalogenase gene sequence from $B$. megaterium WSH-002 was further analysed to identify and predict the protein family domain using InterPro Scan online software (www.ebi.ac.uk/interpro/) [43].

\section{Results and Discussions}

\section{Screening for Putative Haloacid Dehalogenase Gene from B. megaterium WSH-002}

The annotated genome was downloaded as an xls file and the process of screening the possible putative haloacid dehalogenase sequence was done manually. In total, at least 12 possible putative haloacid dehalogenase genes were found in the genome, as shown in Table 1. The longest sequence was gene locus BMWSH_2800 (789 bp) and the shortest BMWSH_2297 (270 bp).

All twelve genes were BLASTn using NCBI databases. It was found that only 6 gene sequences showed the highest identity to dehalogenase genes, BMWSH_1544 (97.6\%), BMWSH_2800 (99.2\%),
BMWSH_4074 (99.6\%), BMWSH_4378 (98.2\%) and BMWSH_4521 (99.5\%) as shown in Table 2. BMWSH_1544 (97.6\%) showed that B. megaterium WSH-002 has highly similar to $B$. megaterium ATCC 12872/QMB1551 since most of the gene sequences found in B. megaterium WSH-002 was homologous to $B$. megaterium ATCC12872/QMB1551 [44].

Meanwhile, there were several gene loci of putative haloacid dehalogenase that have shown results of other than dehalogenases (BMWSH_0693, BMWSH_0746, BMWSH_1820, BMWSH_2297, BMWSH_4020) (Table 2). For example, gene locus BMWSH_0693 has a high similarity to HD domain-containing protein (98.4\%). This domain was classified as a superfamily that contributes the major functionality of phosphohydrolase which catalyses both metal dependant and independent phosphomonoesterase and phosphodiesterase reactions for various ranges of substrates including CCA-adding enzymes, uridylyl transferases dGTPase, polyA polymerases, and stringent-response guanosine polyphosphate hydrolase [45, 46].

Table 1. List of all possible putative haloacid dehalogenase genes in the genome Bacillus megaterium WSH002

\begin{tabular}{|c|c|c|c|c|c|}
\hline No. & Gene Product & $\begin{array}{l}\text { CDS Location } \\
\text { (bp) }\end{array}$ & Gene Locus ID & $\begin{array}{l}\text { Strand } \\
(+/-)\end{array}$ & $\begin{array}{l}\text { Length } \\
\text { (bp) }\end{array}$ \\
\hline 1. & $\begin{array}{l}\text { Hydrolase (HAD superfam- } \\
\text { ily), YqeK }\end{array}$ & 685729_686301 & BMWSH_0693 & + & 573 \\
\hline 2. & $\begin{array}{l}\text { HAD-superfamily hydro- } \\
\text { lase-like protein }\end{array}$ & 735987_735412 & BMWSH_0746 & - & 576 \\
\hline 3. & $\begin{array}{l}\text { Hydrolase, haloacid dehalo- } \\
\text { genase-like family }\end{array}$ & 1473045_1473788 & BMWSH_1544 & + & 744 \\
\hline 4. & $\begin{array}{l}\text { HAD-superfamily hydro- } \\
\text { lase, subfamily IIB }\end{array}$ & 1698317_1697544 & BMWSH_1820 & - & 774 \\
\hline 5. & $\begin{array}{l}\text { Hydrolase, HAD superfam- } \\
\text { ily }\end{array}$ & 2061582_2061313 & BMWSH_2297 & - & 270 \\
\hline 6. & $\begin{array}{l}\text { 2-haloalkanoic acid dehalo- } \\
\text { genase (EC 3.8.1.2) }\end{array}$ & 2289639_2290310 & BMWSH_2542 & + & 672 \\
\hline 7. & $\begin{array}{l}\text { 2-haloalkanoic acid dehalo- } \\
\text { genase (EC 3.8.1.2) }\end{array}$ & 2520695_2521483 & BMWSH_2800 & + & 789 \\
\hline 8. & $\begin{array}{l}\text { Hydrolase, haloacid dehalo- } \\
\text { genase-like family protein } \\
\text { BCZK2594 }\end{array}$ & 3349662_3348991 & BMWSH_3713 & - & 672 \\
\hline 9. & $\begin{array}{l}\text { Hydrolase (HAD superfam- } \\
\text { ily) in the cluster with } \\
\text { DUF1447 }\end{array}$ & 3641408_3640626 & BMWSH_4020 & - & 783 \\
\hline 10. & $\begin{array}{l}\text { Hydrolase, haloacid dehalo- } \\
\text { genase-like family }\end{array}$ & 3693107_3693904 & BMWSH_4074 & + & 798 \\
\hline 11. & $\begin{array}{l}\text { 2-haloalkanoic acid dehalo- } \\
\text { genase (EC 3.8.1.2) }\end{array}$ & 3980565_3979906 & BMWSH_4378 & - & 660 \\
\hline 12. & $\begin{array}{l}\text { Hydrolase, haloacid dehalo- } \\
\text { genase-like family }\end{array}$ & 4127620_4126961 & BMWSH_4521 & - & 660 \\
\hline
\end{tabular}




\begin{tabular}{|c|c|c|c|c|}
\hline Gene Locus ID & Protein Name & Species & $\begin{array}{l}\text { Accession } \\
\text { Number }\end{array}$ & $\begin{array}{l}\text { Percentage } \\
\text { Identity }\end{array}$ \\
\hline BMWSH_0693 & $\begin{array}{l}\text { HD domain-containing pro- } \\
\text { tein }\end{array}$ & $\begin{array}{c}\text { Bacillus megaterium } \\
\text { (strain ATCC 12872 / } \\
\text { QMB1551) }\end{array}$ & D5DSX3 & $98.4 \%$ \\
\hline BMWSH_0746 & Nucleotidase & $\begin{array}{c}\text { Bacillus megaterium } \\
\text { (strain ATCC 12872 / } \\
\text { QMB1551) }\end{array}$ & D5DSD5 & $99.0 \%$ \\
\hline BMWSH_1544 & $\begin{array}{l}\text { Haloacid dehalogenase-like } \\
\text { hydrolase }\end{array}$ & $\begin{array}{c}\text { Bacillus megaterium } \\
\text { (strain ATCC 12872 / } \\
\text { QMB1551) }\end{array}$ & D5E2W1 & $97.6 \%$ \\
\hline BMWSH_1820 & $\begin{array}{l}\text { HAD-superfamily hydro- } \\
\text { lase, subfamily IIB }\end{array}$ & $\begin{array}{c}\text { Bacillus megaterium } \\
\text { (strain ATCC 12872 / } \\
\text { QMB1551) }\end{array}$ & D5DZR9 & $88.7 \%$ \\
\hline BMWSH_2297 & $\begin{array}{l}\text { Cys_rich_CPCC domain- } \\
\text { containing protein }\end{array}$ & $\begin{array}{c}\text { Bacillus megaterium } \\
\text { (strain ATCC 12872 / } \\
\text { QMB1551) }\end{array}$ & D5DW41 & $97.8 \%$ \\
\hline BMWSH_2542 & $\begin{array}{c}\text { L-2-haloalkanoic acid } \\
\text { dehalogenase }\end{array}$ & Bacillus sp. AFS018417 & A0A2A8S566 & $67.4 \%$ \\
\hline BMWSH_2800 & $\begin{array}{l}\text { HAD-superfamily hydro- } \\
\text { lase, subfamily IA, variant } 3\end{array}$ & $\begin{array}{c}\text { Bacillus megaterium } \\
\text { (strain ATCC 12872 / } \\
\text { QMB1551) }\end{array}$ & D5DTA0 & $99.2 \%$ \\
\hline BMWSH_3713 & $\begin{array}{l}\text { Haloacid dehalogenase-like } \\
\text { hydrolase domain protein }\end{array}$ & $\begin{array}{c}\text { Bacillus megaterium } \\
\text { (strain ATCC 12872 / } \\
\text { QMB1551) }\end{array}$ & D5E2P3 & $95.1 \%$ \\
\hline BMWSH_4020 & Cof-like hydrolase & $\begin{array}{c}\text { Bacillus megaterium } \\
\text { (strain ATCC 12872 / } \\
\text { QMB1551) }\end{array}$ & D5E0B2 & $98.8 \%$ \\
\hline BMWSH_4074 & $\begin{array}{l}\text { HAD-superfamily hydro- } \\
\text { lase, subfamily IIB }\end{array}$ & $\begin{array}{c}\text { Bacillus megaterium } \\
\text { (strain ATCC 12872 / } \\
\text { QMB1551) }\end{array}$ & D5E062 & $99.6 \%$ \\
\hline BMWSH_4378 & $\begin{array}{l}\text { Haloacid dehalogenase, type } \\
\text { II }\end{array}$ & $\begin{array}{c}\text { Bacillus megaterium } \\
\text { (strain ATCC 12872 / } \\
\text { QMB1551) }\end{array}$ & D5E2F6 & $98.2 \%$ \\
\hline BMWSH_4521 & $\begin{array}{l}\text { Haloacid dehalogenase-like } \\
\text { hydrolase family protein }\end{array}$ & $\begin{array}{c}\text { Bacillus megaterium } \\
\text { (strain ATCC 12872 / } \\
\text { QMB1551) }\end{array}$ & D5E193 & $99.5 \%$ \\
\hline
\end{tabular}

Besides that, gene locus BMWSH_0746 also has a high similarity to nucleotidase (99.0\%). This protein consists of a domain that is remotely related to HAD-like hydrolase domains and SCOP HAD-like hydrolase domain superfamily. This enzyme has a significant role in the digestion of nucleic acids since it catalyses the hydrolysis of a nucleotide into a nucleoside and a phosphate [47]. For instance, the conversion of adenosine monophosphate to adenosine. Most bacteria can utilize nucleotides as sources of purines or pyrimidines but these have to be dephosphorylated by extracellular nucleotidases before entering the cell [48].

According to Table 2, gene locus BMWSH_2297 is similar to cysteine-rich CPCC domain protein (97.8\%). This protein family was identified as an uncharacterised functional protein although it can be found in bacteria, archaea, eukaryotes, and viruses. This type of domain constitutes six conserved cysteines and a conserved CPCC sequence motif. In contrast, the gene locus BMWSH_4020 is highly similar to Cof-like hydrolase [49]. Cof-like hydrolase associated with dehalogenase from Enterobacter allows this bacterium to grow on a medium containing halogenated compound as the sole carbon source [49]. However, the specialized function of this protein remains obscure. Interestingly, gene locus BMWSH_2542 has a similarity to L-2-haloalkanoic acid dehalogenase belongs to Bacillus sp. AFS018417 [50] although the percentage of protein identity is insignificant. L-2-haloalkanoic acid dehalogenase is common among many bacteria species but not D-2-haloalkanoic acid dehalogen 
Table 3. The prediction of protein family classification and their molecular function

\begin{tabular}{|c|c|c|c|c|}
\hline \multirow{2}{*}{ Gene Locus ID } & \multicolumn{2}{|c|}{ Protein Family } & \multicolumn{2}{|c|}{ Molecular Function } \\
\hline & $\begin{array}{l}\text { Accession } \\
\text { Number }\end{array}$ & Classification & $\begin{array}{l}\text { Accession } \\
\text { Number }\end{array}$ & Description \\
\hline BMWSH_1544 & IPR041492 & $\begin{array}{l}\text { Haloacid dehalogenase- } \\
\text { like hydrolase }\end{array}$ & - & - \\
\hline BMWSH_2800 & & Phosphoserine phospha- & GO:0016787 & hydrolase activity \\
\hline & IPRU44266 & tase YsaA & GO:0004647 & $\begin{array}{l}\text { phosphoserine phos- } \\
\text { phatase activity }\end{array}$ \\
\hline BMWSH_3713 & IPR041492 & $\begin{array}{l}\text { Haloacid dehalogenase- } \\
\text { like hydrolase }\end{array}$ & - & 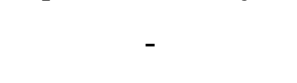 \\
\hline BMWSH_4074 & IPR000150 & Cof family & GO:0016787 & hydrolase activity \\
\hline & & & GO:0016787 & hydrolase activity \\
\hline BMWSH_4378 & IPR006328 & $\begin{array}{l}\text { L-2-Haloacid dehalogen- } \\
\text { ase }\end{array}$ & GO:0019120 & $\begin{array}{l}\text { hydrolase activity, } \\
\text { acting on acid halide } \\
\text { bonds, in C-halide } \\
\text { compounds }\end{array}$ \\
\hline BMWSH_4521 & IPR006439 & $\begin{array}{c}\text { HAD hydrolase, subfam- } \\
\text { ily IA }\end{array}$ & GO:0016787 & hydrolase activity \\
\hline
\end{tabular}

**The following information was retrieved from InterPro databases

ases [51, 52].

\section{Further analysis of gene locus with putative dehalogenase genes}

The six highest percentage of gene locus was further analysed and summarised shown in Table 3. The gene locus BMWSH_1544 and BMWSH_3713 were classified as haloacid dehalogenase-like hydrolase (IPR041492). This protein belongs to the large superfamily of diverse enzymes that catalyse carbon or phosphoryl group transfer reaction on a wide range of substrates using aspartate as an active site in nucleophilic catalysis [53]. However, their molecular function was unable to predict. Gene locus BMWSH_4074 and BMWSH_4521 were known as Cof family (IPR000150) and HAD hydrolase, subfamily IA (IPR006439). Both gene loci showed the same molecular function, which has hydrolase activity (GO:0016787), catalyzing the hydrolysis of various bonds such as C-O, C-N, C-C, phosphoric anhydride bonds [54, 55] but did not predict any specialized functions of the proteins. Furthermore, gene locus BMWSH_2800 was classified as phosphoserine phosphatase YsaA (IPR044266) which has the function of hydrolase specifically acting on ester bonds in the phosphate-containing compound which catalyses the dephosphorylation reaction of phosphoserine yielding serine and phosphate (GO:0004647) [56, 57]. However, its functional properties were significantly different from haloacid dehalogenases. The gene locus BMWSH_4378 was classified as haloacid dehalogenase type II because of the presence of conserved domain HAD_L2-DEX (cd02588) and HAD_type_II (TIGR01428) found in putative haloacid dehalogenase protein sequence (Table 4). According to Marchler-Bauer et al. [58], the domain HAD_type_II in Conserved Domain Databases (CDD) was classified as 2-haloalkanoic acid dehalogenase type II which catalyses the hydrolytic dehalogenation of L-2-haloalkanoic acids to produce the corresponding D-2-hydroxyalkanoic acids [58, 59]. This domain belongs to HAD superfamily of aspartate-nucleophile hydrolase [60]. This domain was also conserved in DhlB and Hdl IVa from Xanthobacter autotrophicus GJ10 and Burkholderia cepacia MBA4 respectively [61, 62]. The HAD_L2-DEX domain in the CDD was classified as L-2-haloacid dehalogenase and has demonstrated several features including active sites, homodimers, and HAD signature motifs. The conserved amino acid residue that acts as an active site nucleophile was aspartate residue (Asp8) found in DhlB and L-DEX from X. autotrophicus and Pseudomonas sp. YL respectively $[63,64]$. Upon the attack of the substrate, this residue forms an ester intermediate and is subsequently hydrolysed by a water molecule [65]. The members of the L-DEX family have been reported 
Table 4. The domain of 2-haloalkanoic acid dehalogenase protein gene BMWSH_4378 of B. megaterium WSH-002

\begin{tabular}{|c|c|c|c|}
\hline Accession Number & Classification of Protein Families & Short Name & $\begin{array}{l}\text { Contributing Member } \\
\text { Database Entries }\end{array}$ \\
\hline IPR006439 & $\begin{array}{l}\text { HAD hydrolase, subfamily IA } \\
\text { (superfamily) }\end{array}$ & HAD-SF_hydro_IA & InterPro \\
\hline TIGR01493 & $\begin{array}{l}\text { HAD hydrolase, family IA, variant } 2 \\
\text { (subfamily) }\end{array}$ & $H A D-S F-I A-v 2$ & TIGRFAMS \\
\hline TIGR01549 & $\begin{array}{l}\text { HAD hydrolase, family IA, variant } 1 \\
\text { (subfamily) }\end{array}$ & $H A D-S F-I A-v 1$ & TIGRFAMS \\
\hline PR00413 & $\begin{array}{c}\text { HAD family } \\
\text { (family) }\end{array}$ & HADHALOGNASE & PRINTS \\
\hline IPR006328 & $\begin{array}{l}\text { L-2-Haloacid dehalogenase } \\
\text { (superfamily) }\end{array}$ & 2-HAD & InterPro \\
\hline cd02588 & $\begin{array}{l}\text { L-2-haloacid dehalogenase } \\
\text { (domain) }\end{array}$ & $H A D \_L 2-D E X$ & CDD \\
\hline TIGR01428 & $\begin{array}{l}\text { haloacid dehalogenase, type II } \\
\text { (family) }\end{array}$ & HAD_type_II & TIGRFAMS \\
\hline IPR041492 & $\begin{array}{l}\text { Haloacid dehalogenase-like hydrolase } \\
\text { (superfamily) }\end{array}$ & $H A D \_2$ & InterPro \\
\hline PF13419 & $\begin{array}{l}\text { Haloacid dehalogenase-like hydrolase } \\
\text { (family) }\end{array}$ & $H A D \_2$ & Pfam \\
\hline IPR023214 & $\begin{array}{c}\text { HAD superfamily } \\
\text { (superfamily) }\end{array}$ & $H A D \_s f$ & InterPro \\
\hline G3DSA:3.40.50.1000 & $\begin{array}{l}\text { HAD superfamily/HAD-like } \\
\text { (Homologous superfamily) }\end{array}$ & - & CATH-Gene3D \\
\hline IPR023198 & $\begin{array}{l}\text { Phosphoglycolate phosphatase-like, } \\
\text { domain } 2 \\
\text { (domain) }\end{array}$ & PGP-like_dom2 & InterPro \\
\hline G3DSA:1.10.150.240 & $\begin{array}{l}\text { Putative phosphatase; domain } 2 \\
\text { (Homologous superfamily) }\end{array}$ & - & CATH-Gene3D \\
\hline IPR036412 & $\begin{array}{l}\text { HAD-like superfamily } \\
\text { (superfamily) }\end{array}$ & HAD-like_sf & InterPro \\
\hline SSF56784 & $\begin{array}{l}\text { HAD-like } \\
\text { (Homologous superfamily) }\end{array}$ & - & SUPERFAMILY \\
\hline SFLDF00045 & $\begin{array}{l}\text { 2-haloacid dehalogenase } \\
\text { (family) }\end{array}$ & 2-haloacid_dehalogenase & SFLD \\
\hline SFLDG01135 & $\begin{array}{l}\text { C1.5.6: HAD, Beta-PGM, Phospha- } \\
\text { tase Like } \\
\text { (family) }\end{array}$ & $\begin{array}{l}\text { C1.5.6:_HAD_Beta- } \\
\text { PGM_Phospha }\end{array}$ & SFLD \\
\hline PTHR43316 & $\begin{array}{l}\text { Hydrolase, Haloacid Dehalogenase- } \\
\text { Related } \\
\text { (family) }\end{array}$ & - & PANTHER \\
\hline PTHR43316: SF3 & $\begin{array}{c}\text { Haloacid Dehalogenase, Type II } \\
\text { (AFU_ORTHOLOGUE } \\
\text { AFUA_2G07750)-Related } \\
\text { (family) }\end{array}$ & - & PANTHER \\
\hline
\end{tabular}

to occur as homodimers including L-DEX from Pseudomonas sp. YL [66]. The dimer of DhlB is more tightly packed compared to L-DEX due to the absence of a small atrium subdomain that provides a major contribution to the dimer interface [63]. Most of the amino acid residues involved in dimerization are conserved in the family of L-2haloacid dehalogenase [67]. Therefore, this study suggested that predicted domains found in gene locus BMWSH_4378 contribute to the functional properties of haloacid dehalogenase type II that exhibit the function of hydrolase specifically acting on acid halide bonds in halogenated compounds [68]. Due to this evidence, the gene locus BMWSH_4378 as putative haloacid dehalogenase was subjected to future studies for its structure and functions.

\section{Conclusion}

DNA annotation or genome annotation allows the identifying the locations of genes and all of the coding regions in a genome and determining what 
those genes do. Here, putative dehalogenases were identified from the whole genome studies in $B$. megaterium WSH-002. It was curious why a single bacterium may have more than one dehalogenase with similar or different functions. However, the presence of dehalogenase regulatory and uptake genes was not detected. This study allows researchers to study not only the genes which code for the important proteins that keep the cell to survive in the highly contaminated area but also the regions of the DNA that have other important roles, such as the regulation of the specific gene(s) particularly dehalogenases need to be investigated.

\section{Acknowledgement}

The authors would like to express their appreciation and gratitude to FRGS (Ministry of Higher Education R.J130000.7854.5F189/ FRGS/1/2019/STG05/UTM/01/1) for financial assistance.

\section{References}

1. Fowden L (1968) The occurrence and metabolism of carbon-halogen compounds, Proceedings of the Royal Society of London. Series B. Biological Sciences 171 (1022): 5-18. doi: 10.1098/rspb.1968.0052.

2. Gribble GW (2003) The diversity of naturally produced organohalogens. Chemosphere 52 (2): 289-297. doi: 10.1016/S0045-6535(03)00207-8.

3. Häggblom MM, Bossert ID (2004) Halogenated organic compounds-a global perspective, in Dehalogenation, Springer. p. 3-29.

4. O’Brien D, Lewis S, Gallen C et al. (2014) Barron River pesticide monitoring and Cairns WWTP WQ assessment, Centre for Tropical Water \& Aquatic Ecosystem Research (TropWATER) Publication, James Cook University, Cairns, Australia, 49.

5. Saari LL, Mauvais CJ (2018) Sulfonylurea herbicideresistant crops, in Herbicide-resistant crops. CRC Press. 127-142.

6. Plewa MJ, Simmons JE, Richardson SD, Wagner ED (2010) Mammalian cell cytotoxicity and genotoxicity of the haloacetic acids, a major class of drinking water disinfection by-products. Environmental and molecular mutagenesis 51 (8-9): 871-878. doi: 10.1002/em.20585.

7. Prüss-Ustün A, Vickers C, Haefliger P, Bertollini R (2011) Knowns and unknowns on burden of disease due to chemicals: a systematic review. Environmental Health 10 (1): 1-15. doi: 10.1186/1476-069x-10-9.

8. Kemf E (2013) GCO--Global Chemicals Outlook: Towards Sound Management of Chemicals. United Nations Environment Programme.
9. Awang RA, Latiff, Majid M, Dzulkifli A (2011) Case study: Malaysian information service on pesticide toxicity, Encyclopaedia of occupational health and safety. Geneva, Switzerland: International Labour Organization, 22.

10. Gribble GW (2015) A recent survey of naturally occurring organohalogen compounds. Environmental Chemistry 12 (4): 396-405. doi:10.1071/EN15002.

11. Oyewusi HA, Wahab RA, Huyop F (2020) Dehalogenase-producing halophiles and their potential role in bioremediation. Marine Pollution Bulletin 160: 111603. doi:10.1016/j.marpolbul.2020.111603.

12. Zakary S, Oyewusi HA, Huyop F (2020) Dehalogenases for pollutant degradation: A mini-review. Journal Tropical Life Science 11 (1): 17-24. doi:10.11594/jtls.11.01.03.

13. Kirkinci SF, Edbeib MF, Aksoy HM et al. (2021) Identification of Dalapon degrading bacterial strain, Psychrobacter sp. TaeBurcu001 isolated from Antarctica. Polar Science 28: 100656. doi:10.1016/j.polar.2021.100656.

14. Slater JH, Bull AT, Hardman DJ (1996) Microbial dehalogenation of halogenated alkanoic acids, alcohols and alkanes. Advances in microbial physiology 38: 133176. doi:10.1016/S0065-2911(08)60157-5.

15. Adamu A, Wahab RA, Aliyu F et al. (2020) Haloacid dehalogenases of Rhizobium sp. and related enzymes: Catalytic properties and mechanistic analysis. Process Biochemistryl 92: 437-446. doi:10.1016/j.procbio.2020.02.002.

16. Kerr LM, Marchesi JR (2006) Isolation of novel bacteria able to degrade $\alpha$-halocarboxylic acids by enrichment from environmental samples. Chemosphere 64 (5): 848855. doi:10.1016/j.chemosphere.2005.10.042.

17. Kwok SY, Siu AFM, Ngai SM et al. (2007) Proteomic analysis of Burkholderia cepacia MBA4 in the degradation of monochloroacetate. Proteomics 7 (7): 1107-1116. doi:10.1002/pmic.200600660.

18. Vary PS, Biedendieck R, Fuerch T et al. (2007) Bacillus megaterium-from simple soil bacterium to industrial protein production host. Applied Microbiology and Biotechnology 76 (5): 957-967. doi:10.1007/s00253007-1089-3.

19. Akhtar P, Anand SP, Watkins SC, Khan SA (2009) The tubulin-like RepX protein encoded by the pXO1 plasmid forms polymers in vivo in Bacillus anthracis. Journal of $\begin{array}{llll}\text { Bacteriology } & 191 & \text { (8): } 2493-2500 .\end{array}$ doi:10.1128/JB.00027-09.

20. Lioy VS, Rey O, Balsa D et al. (2010) A toxin-antitoxin module as a target for antimicrobial development. Plasmid $63 \quad$ (1): 31-39. doi: 10.1016/j.plasmid.2009.09.005.

21. Aksoy HM, Akça İ, Kaya Y, Ozturk M (2015) Isolation and Characterization of Bacillus thuringiensis Isolated from Soil and their Possible Impact on Culex pipiens. 
Egyptian Journal of Biological Pest Control 25 (2): 439444. doi:10.81043/aperta.81035.

22. Aksoy HM, Saruhan I, Yılmaz K, Öztürk M (2018) Morphological changes caused by Bacillus megaterium on adult emergence of fall webworm's pupa, Hyphantria cunea (Drury)(Lepidoptera: Erebidae). Journal of

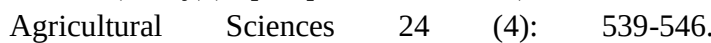
doi:10.15832/ankutbd.349474.

23. Al-Gheethi ASS (2015) Recycling of sewage sludge as production medium for cellulase by a Bacillus megaterium strain. International Journal of Recycling of Organic Waste in Agriculture 4 (2): 105-119. doi:10.1007/s40093-015-0090-6.

24. Luo LZ, Zhao X, Huang X et al. (2016), Isolation, identification, and optimization of culture conditions of a bioflocculant-producing bacterium Bacillus megaterium SP1 and its application in aquaculture wastewater treatment. BioMed research international 2016. doi: 10.1155/2016/2758168.

25. Dobrzanski T, Gravina F, Steckling B et al. (2018) Bacillus megaterium strains derived from water and soil exhibit differential responses to the herbicide mesotrione. PloS One 13 (4): e0196166. doi:10.1371/journal.pone.0196166.

26. Akcay K, Kaya Y (2019) Isolation, characterization and molecular identification of a halotolerant Bacillus megaterium CTBmeg1 able to grow on halogenated compounds. Biotechnology \& Biotechnological Equipment $\quad 33 \quad$ (1): $\quad 945-953$. doi:10.1080/13102818.2019.1631717.

27. Wahhab BHA, Anuar NFSK, Wahab RA et al. (2020) Identification and characterization of a 2, 2dichloropropionic acid (2, 2-DCP) degrading alkalotorelant bacterium strain BHS1 isolated from Blue Lake, Turkey. Journal of Tropical Life Science 10 (3): 245-252. doi: 10.11594/jtls.10.03.08.

28. Gordon S (2002) Genomics and World Health. Report of the Advisory Committee on Health Research. Geneva: World Health Organization, 2002. ISBN 92-4-154554-2. Transactions of The Royal Society of Tropical Medicine and Hygiene 96 (6): 669-669. doi:10.1016/s00359203(02)90347-0.

29. Wasmund K, Pelikan C, Schintlmeister A et al. (2021) Genomic insights into diverse bacterial taxa that degrade extracellular DNA in marine sediments. Nature Microbiology: 1-14. doi:10.1038/s41564-021-00917-9.

30. White O, Eisen JA, Heidelberg JF et al. (1999) The Genome sequence of the radioresistant bacterium Deinococcus radiodurans R1. Science 286 (5444): 1571 1577. doi:10.1126/science.286.5444.1571.

31. Kunst F, Ogasawara N, Moszer I et al. (1997) The complete genome sequence of the gram-positive bacterium Bacillus subtilis. Nature 390 (6657): 249-256. doi:10.1038/36786.
32. Goodner BW, Markelz BP, Flanagan MC et al. (1999) Combined genetic and physical map of the complex genome of Agrobacterium tumefaciens. Journal of Bacteriology $181 \quad$ (17): $\quad 5160-5166$. doi:10.1128/JB.181.17.5160-5166.1999.

33. Medema MH, de Rond T, Moore BS (2021) Mining genomes to illuminate the specialized chemistry of life. Nature Reviews Genetics: 1-19. doi:10.1038/s41576021-00363-7.

34. Chandran H, Meena M, Sharma K, (2020) Microbial biodiversity and bioremediation assessment through omics approaches. Frontiers in Environmental Chemistry 1: 9. doi:10.3389/fenvc.2020.570326.

35. Singh AK, Bilal M, Iqbal HM, Raj A (2021) Trends in predictive biodegradation for sustainable mitigation of environmental pollutants: Recent progress and future outlook. Science of The Total Environment: 144561. doi:10.1016/j.scitotenv.2020.144561.

36. Chan WY, Wong M, Guthrie J et al. (2010) Sequenceand activity-based screening of microbial genomes for novel dehalogenases. Microbial Biotechnology 3 (1): 107-120. doi:10.1111/j.1751-7915.2009.00155.x.

37. Oyewusi HA, Wahab RA, Huyop F (2021) Whole genome strategies and bioremediation insight into dehalogenase-producing bacteria, Molecular Biology Reports: 1-15. doi:10.1007/s11033-021-06239-7.

38. Wahhab BHA, Samsulrizal NH, Edbeib MF et al. (2021) Genomic analysis of a functional haloacid-degrading gene of Bacillus megaterium strain BHS1 isolated from Blue Lake (Mavi Gölü, Turkey). Annals of Microbiology 71 (1): 1-11. doi:10.1186/s13213-02101625-9.

39. Liu L, Li Y, Zhang J, Zou W et al. (2011) Complete genome sequence of the industrial strain Bacillus megaterium WSH-002. Journal of Bacteriology, 193(22): 6389-6390.10.1128/JB.06066-11.

40. Overbeek R, Olson R, Pusch GD et al. (2014) The SEED and the Rapid Annotation of microbial genomes using Subsystems Technology (RAST). Nucleic Acids Research $42 \quad$ (D1): $\quad$ D206-D214. doi:10.1093/nar/gkt1226.

41. Altschul SF, Madden TL, Schäffer AA et al. (1997) Gapped BLAST and PSI-BLAST: a new generation of protein database search programs. Nucleic Acids Research 25 (17): 3389-3402. doi: 10.1093/nar/25.17.3389.

42. Pundir S, Martin MJ, O’Donovan (2017) UniProt protein knowledgebase, in Protein Bioinformatics, Springer. p. 41-55.

43. Mitchell A, Chang HY, Daugherty L et al. (2015) The InterPro protein families database: the classification resource after 15 years. Nucleic Acids Research 43 (D1): D213-D221. doi:10.1093/nar/gku1243.

44. Eppinger M, Bunk B, Johns MA et al. (2011) Genome sequences of the biotechnologically important Bacillus 
megaterium strains QM B1551 and DSM319. Journal of Bacteriology $\quad 193 \quad$ (16): $\quad 4199-4213$. doi:10.1128/JB.00449-11.

45. Aravind L, Koonin EV (1998) The HD domain defines a new superfamily of metal-dependent phosphohydrolases. Trends in Biochemical Sciences 23 (12): 469-472. doi:10.1016/S0968-0004(98)01293-6.

46. Zimmerman MD, Proudfoot M, Yakunin A, Minor W (2011), Structural Insight into the Mechanism of Substrate Specificity and Catalytic Activity of an HDDomain Phosphohydrolase: The 5; \# 8242; Deoxyribonucleotidase YfbR from Escherichia coli. Journal of Molecular Biology 378 (1); 04, 2008) doi:10.1016/j.jmb.2008.02.036.

47. Zimmermann H, Zebisch M, Sträter N (2012) Cellular function and molecular structure of ecto-nucleotidases. $\begin{array}{llll}\text { Purinergic Signalling } 8 & \text { (3): 437-502. }\end{array}$ doi:10.1007/s11302-012-9309-4.

48. Kilstrup M, Hammer K, Jensen PR, Martinussen J (2005) Nucleotide metabolism and its control in lactic acid bacteria. FEMS Microbiology Reviews 29 (3): 555590. doi:10.1016/j.fmrre.2005.04.006.

49. Nemati M, Abdulghader MF, Gicana RG et al. (2013) Identification of putative Cof-like hydrolase associated with dehalogenase in Enterobacter cloacae MN1 isolated from the contaminated sea-side area of the Philippines. Malaysian Journal of Microbiology 9 (3): 253-259.

50. Gillis A, Fayad N, Makart L et al. (2018) Role of plasmid plasticity and mobile genetic elements in the entomopathogen Bacillus thuringiensis serovar israelensis. FEMS Microbiology Reviews 42 (6): 829856. doi:10.1093/femsre/fuy034.

51. Sudi IY, Hamid AAA, Shamsir MS et al. (2014) Insights into the stereospecificity of the d-specific dehalogenase from Rhizobium sp. RC1 toward d-and l-2chloropropionate. Biotechnology \& Biotechnological $\begin{array}{llll}\text { Equipment } & 28 & \text { (4): }\end{array}$ doi:10.1080/13102818.2014.937907.

52. Sudi IY, Shamsir MS, Jamaluddin H et al. (2014) Interactions of non-natural halogenated substrates with D-specific dehalogenase (DehD) mutants using in silico studies. Biotechnology \& Biotechnological Equipment 28 (5): 949-957. doi:10.1080/13102818.2014.960663.

53. Seifried A, Schultz J, Gohla A (2013) Human HAD phosphatases: structure, mechanism, and roles in health and disease. The FEBS Journal 280 (2): 549-571. doi:10.1111/j.1742-4658.2012.08633.x.

54. Cornish-Bowden A (2014) Current IUBMB recommendations on enzyme nomenclature and kinetics. Perspectives in Science 1 (1-6): 74-87. doi:10.1016/j.pisc.2014.02.006.

55. Schomburg I, Chang A, Schomburg D (2014) Standardization in enzymology - Data integration in the world' s enzyme information system BRENDA.
Perspectives in Science 1 (1-6): 15-23. doi:10.1016/j.pisc.2014.02.002.

56. Wang W, Kim R, Jancarik J, Yokota H, Kim SH (2001) Crystal structure of phosphoserine phosphatase from Methanococcus jannaschii, a hyperthermophile, at $1.8 \AA$ resolution. Structure 9 (1): 65-71. doi:10.1016/S09692126(00)00558-X.

57. Chiba Y, Horita S, Ohtsuka J, Arai H et al. (2013) Structural units important for activity of a novel-type phosphoserine phosphatase from Hydrogenobacter thermophilus TK-6 revealed by crystal structure analysis. Journal of Biological Chemistry 288 (16): 11448-11458. doi:10.1074/jbc.M112.449561.

58. Marchler-Bauer A, Bo Y, Han L et al. (2017),m CDD/SPARCLE: functional classificationbof proteins via subfamily domain architectures. Nucleic Acids $\begin{array}{llll}\text { Research } & 45 & \text { (D1): } & \text { D200-D203. }\end{array}$ doi:10.1093/nar/gkw1129.

59. Janssen DB, Oppentocht JE, Poelarends GJ, (2001) Microbial dehalogenation. Current Opinion in Biotechnology 12 (3): 254-258. doi:10.1016/S09581669(00)00208-1.

60. Hill KE., Marchesi JR, Weightman AJ (1999) Investigation of two evolutionarily unrelated halocarboxylic acid dehalogenase gene families. Journal $\begin{array}{llll}\text { of } & \text { Bacteriology } & 181 & \text { (8): } \quad 2535-2547 .\end{array}$ doi:10.1128/JB.181.8.2535-2547.1999.

61. Van Der Ploeg, van Hall JG, Janssen DB (1991) Characterization of the haloacid dehalogenase from Xanthobacter autotrophicus GJ10 and sequencing of the dhlB gene. Journal of Bacteriology 173 (24): 7925-7933. doi:10.1128/jb.173.24.7925-7933.1991.

62. Tsang JS, Sam L (1999) Cloning and characterization of a cryptic haloacid dehalogenase from Burkholderia cepacia MBA4. Journal of Bacteriology 181 (19): 60036009. doi:10.1128/JB.181.19.6003-6009.1999.

63. Ridder IS, Rozeboom HJ, Kalk KH et al. (1997) Threedimensional structure of L-2-haloacid dehalogenase from Xanthobacter autotrophicus GJ10 complexed with the substrate-analogue formate. Journal of Biological $\begin{array}{lll}\text { Chemistry } & 272 \quad \text { (52): } \quad 33015-33022 .\end{array}$ doi:10.1074/jbc.272.52.33015.

64. Hisano T, Hata Y, Fujii T et al. (1996) Crystal Structure of L-2-Haloacid Dehalogenase from Pseudomonas sp. YL: an $\alpha / \beta$ Hydrolase Structure that is Different from the $\alpha / \beta$ Hydrolase Fold. Journal of Biological Chemistry 271 (34): 20322-20330. doi:10.1074/jbc.271.34.20322.

65. Liu TK, Miyagi M, Tsunasawa S et al. (1997) Paracatalytic inactivation of L-2-haloacid dehalogenase from Pseudomonas sp. YL by hydroxylamine: evidence for the formation of an ester intermediate. Journal of Biological Chemistry 272 (6): 3363-3368. doi:10.1074/jbc.272.6.3363.

66. Fetzner S, Lingens F (1994) Bacterial dehalogenases: biochemistry, genetics, and biotechnological 
applications. Microbiological Reviews 58 (4): 641-685. doi:10.1128/mr.58.4.641-685.1994.

67. Kurihara T, Liu JQ, Nardi-Dei V et al. (1995) Comprehensive site-directed mutagenesis of L-2-halo acid dehalogenase to probe catalytic amino acid residues.
The Journal of Biochemistry 117 (6): 1317-1322. doi:10.1093/oxfordjournals.jbchem.a124861.

68. Wang G, Li R, Li S, Jiang J, (2010) A novel hydrolytic dehalogenase for the chlorinated aromatic compound chlorothalonil. Journal of Bacteriology 192 (11): 27372745. doi:10.1128/JB.01547-09. 